\title{
Obtención del Enunciado de un Problema Investigando su Solución
}

\author{
Sebastião R. Ferreira \\ Universidad Federal de Río Grande del Norte, Departamento de Ingeniería Química, Av. Salgado \\ Filho 3000, 59078-970 Natal,RN-Brasil (e-mail: ferreira@eq.ufrn.br, seba@ufrnet.br)
}

\begin{abstract}
Resumen
En este trabajo se presenta un método de enseñanza-aprendizaje que consiste en que los estudiantes de una disciplina aprendan a encontrar el enunciado de un problema partiendo de su solución. A un grupo de estudiantes se les presentó la solución de un problema de intercambiadores de calor y se les solicitó que lo analizasen y escribiesen un enunciado para el problema. Los estudiantes presentaron enunciados razonables, pero al plantear el problema algunos se preocuparon más por los aspectos teóricos del tema y otros por los prácticos. Ellos se olvidaron de rehacer los cálculos empleados para seleccionar los intercambiadores, lo que podría haber modificado la elección de determinado intercambiador. Se concluye que la metodología es adecuada para ser empleada en la enseñanza de transferencia de calor, representando una herramienta para ampliar el conocimiento y comprender mejor los conceptos involucrados en el tema del problema.
\end{abstract}

Palabras clave: solución de problemas, planteo de problemas, enseñanza-aprendizaje, intercambiadores de calor

\section{Obtaining the Statement of a Problem Based on its Solution}

\begin{abstract}
In this work a learning-teaching methodology consisting of giving the student the task of finding the statement of a problem based on its solution. The solution of a problem involving heat exchangers was given to a group of students. They were asked to analyze the solution and write a statement for the problem. The students performed well and gave different reasonable statements, but some were more concerned with theoretical aspects and others with practical aspects. They failed to redo the calculations used to select the exchangers, which could have affected the choice of the type of exchanger. It is concluded that the methodology is can be used in teaching heat transfer, representing a tool to widen knowledge and better understand the concepts involved in the subject of the problem.
\end{abstract}

Keywords: problems solution, problems statement, teaching-learning, heat exchangers 


\section{INTRODUCCIÓN}

Hay hechos que sólo pueden ser elucidados, dando marcha atrás o hacia delante, tal como en una pesquisa policial. Si una niña aparece muerta en el jardín de un edificio de departamentos, se conduce una pesquisa para encontrar el o los posibles culpables o bien llegar a otra conclusión, como, por ejemplo, que fue un accidente. Si un auto no arranca a la mañana, cuando alguien intenta salir a trabajar, hay que resolver personalmente el inconveniente o llamar a un mecánico o electricista. Actualmente, en física se lleva a cabo una serie de investigaciones para conocer fenómenos que ocurrieron a millones de años-luz, tales como la desaparición de estrellas. En muchas ocasiones se previene una posible situación problemática, como el derrame o robo de gas o petróleo de tuberías, insertando equipamientos que evalúan la caída de presión en el sistema. En general, esos equipos están conectados a programas computacionales, que realizan por lo menos balances de materia y cantidad de movimiento, y diagnostican si hubo o no falla en el funcionamiento del sistema, para tratar de resolver o remediar el problema. Ésta es una situación especial en la cual se resuelve un problema o un imprevisto, después que él ocurrió, pero con herramientas preparadas con anterioridad.

El diagnóstico de fallas tiene como misión identificar el problema que está afectando a un proceso dado a través del análisis de las señales suministradas por los sensores del proceso (Tarifa y Martínez, 2007). Con el diagnóstico de fallas en plantas químicas se determina, por ejemplo, si hay algún error de operación de la planta, si hay cambios de las corrientes de entrada o salida de productos de la planta etc. Cuando se produce una falla puede aparecer un conjunto finito o infinito de trayectorias o secuencias temporales de datos, que describen las variables del proceso afectadas por dicha falla. Esas secuencias temporales de datos de las variables pueden ser empleadas para identificar o diagnosticar la falla que originó las trayectorias observadas. Las redes neuronales son modelos matemáticos con los que se intenta reproducir la actividad cognitiva del cerebro humano y son empleadas para ayudar en la detección de fallas en plantas, equipos o en diversas otras situaciones. Las redes neuronales deben ser diseñadas y entrenadas para realizar la tarea de diagnóstico y detección de fallas. Lima y Massino (2008), presentan un manual de detección fallas para una planta piloto de elaboración de quesos, desarrollado a partir de los conocimientos de un técnico experto en la monitorización de la planta. Los sistemas expertos son sistemas de información que se originan de conocimientos organizados, de un área específica de la experiencia humana. Los sistemas expertos son desarrollados para resolver algunos problemas, tales como la detección de fallas en procesos industriales, recogiendo la destreza de operadores expertos. Como el término experto es sinónimo de competencia y especialización, el éxito de un sistema experto se debe a que él sólo abarque un campo limitado. Los sistemas expertos suelen aplicarse a sistemas industriales, comúnmente con otras técnicas de inteligencia artificial, tales como redes neuronales y algoritmos genéticos. Según Nurminen et al. (2003), los sistemas expertos son elementos positivos cuando se los entiende como complementos de la tarea de expertos humanos y no como reemplazantes suyos.

Existe una gran cantidad de trabajos en la literatura sobre resolución de problemas (Kantowski, 1980; Pereira, 2002; Polya, 1995; Schoenfeld, 1985), que pueden aportar conocimientos para la elaboración de propuestas para encontrar el enunciado de un problema partiendo de su solución. Por ejemplo, para Wallas (1926), citado por Pereira (2002), las etapas para resolución de problemas son: i) Saturación, en la cual se trabaja en el problema, hasta que tenga sido realizado todo lo que sea posible de ser hecho con él; ii) Incubación, en la cual uno saca el problema del consciente y deja que el subconsciente tome cuenta de él. Es decir, uno duerme con el problema. Ésta es, aparentemente, la etapa fácil de resolución de un problema; iii) Inspiración, en la cual la respuesta del problema llega súbitamente, sin que, aparentemente, uno esté pensando conscientemente en el problema; y iv) Verificación, en la que uno verifica la solución del problema, sólo para tener certeza de que ella está correcta. Para Polya (1995), resolver problemas es una habilidad práctica, como nadar, esquiar o tocar piano, que se puede aprender a través de imitación y práctica. Polya (1995), organizó la resolución de problemas en cuatro etapas: a) Comprender el problema; b) Elaborar un plan de resolución del problema; c) Ejecutar el plan de resolución del problema; y d) Realizar un análisis retrospectivo y verificación de la solución de un 
problema. Cada etapa de resolución de un problema, de (a) a (d), puede ser subdividida en otras que están en la Tabla 1, modificadas y adaptadas de las presentadas en los libros de Dante (2002) y Polya (1995).

Tabla 1: Cómo resolver problemas de matemática partiendo de su enunciado (Dante, 2002; Polya, 1995).

\begin{tabular}{|c|c|}
\hline Etapa & Procedimiento \\
\hline $\begin{array}{l}\text { i) Realizar un análisis del } \\
\text { enunciado de un } \\
\text { problema, tratando de } \\
\text { obtener su solución. }\end{array}$ & $\begin{array}{l}\text { - ¿Qué se pide en el problema? Antes que todo, el enunciado verbal de } \\
\text { un problema debe ser bien comprendido. } \\
\text { - ¿Cuáles son los datos y las condiciones del problema? } \\
\text { - ¿Es posible hacer un esquema, un dibujo o un diagrama, para ayudar } \\
\text { en la resolución del problema? } \\
\text { - ¿Es posible suponer una respuesta al problema? }\end{array}$ \\
\hline $\begin{array}{l}\text { ii) Elaborar un plan para } \\
\text { obtener la solución del } \\
\text { problema a partir de su } \\
\text { enunciado. }\end{array}$ & $\begin{array}{l}\text { - ¿Cuál es el plan elaborado para resolver el problema? Lo principal en } \\
\text { la resolución de un problema es la elaboración del plan de acción. } \\
\text { - ¿Qué estrategias serán empleadas para intentar resolverlo? } \\
\text { - ¿Hay algún problema semejante, que pueda ayudar a resolver éste? } \\
\text { - Acordarse que lo indispensable para la resolución de un problema } \\
\text { matemático es el conocimiento matemático adquirido, por ejemplo, a } \\
\text { través de problemas anteriormente resueltos. }\end{array}$ \\
\hline $\begin{array}{l}\text { iii) Ejecutar el plan para } \\
\text { obtener la solución del } \\
\text { problema. }\end{array}$ & $\begin{array}{l}\text { - Ejecutar el plan elaborado, verificándolo paso a paso. } \\
\text { - Rever la teoría correspondiente al problema a ser resuelto, para } \\
\text { auxiliar en la tarea de obtener una solución. } \\
\text { - El plan es sólo un trayecto general de resolución, pero se necesita } \\
\text { estar convicto de que todos los pasos son correctos. Por eso hay que } \\
\text { examinarlos, uno tras otro, pacientemente, hasta que todo esté } \\
\text { perfectamente claro y que no exista ningún punto nebuloso en el cual } \\
\text { pueda ocultarse algún error. } \\
\text { - Intentar organizar los datos del problema en tablas, diagramas o en } \\
\text { gráficos. } \\
\text { - Tratar de resolver el problema por partes. } \\
\text { - Efectuar los cálculos indicados en el plan, para obtener una solución } \\
\text { del problema. } \\
\text { - Realizar todas las estrategias antes pensadas, obteniendo varias } \\
\text { maneras de resolver el problema. }\end{array}$ \\
\hline $\begin{array}{l}\text { Realizar un análisis } \\
\text { retrospectivo de la } \\
\text { solución del problema, } \\
\text { verificando si a partir } \\
\text { de ella se obtiene el } \\
\text { enunciado planteado. }\end{array}$ & $\begin{array}{l}\text { - ¿Es posible saber si la solución es correcta? } \\
\text { - Un análisis retrospectivo de la resolución, reexaminando el resultado } \\
\text { final y el camino que condujo a éste, puede ayudar a consolidar } \\
\text { conocimientos adquiridos y la capacidad para resolver problemas. } \\
\text { - Rever la solución del problema, contrastándola con el enunciado, } \\
\text { buscando encontrar posibles fallas en ella. } \\
\text { - Verificar los cálculos realizados para obtener la solución, buscando } \\
\text { posibles errores en la metodología de cálculo o en la manera de } \\
\text { plantear la solución. } \\
\text { - ¿Hay otra u otras maneras o métodos para resolver el problema? } \\
\text { - ¿Es posible emplear éste método para resolver otros problemas o } \\
\text { semejantes, a partir de su enunciado? }\end{array}$ \\
\hline
\end{tabular}

Según Polya (1995), la resolución de problemas es un poco de desafío y descubierta, porque no existe un método rígido al cual el estudiante pueda seguir siempre, para encontrar la solución de un problema. Ya para Schoenfeld (1985), la comprensión y enseñanza de matemática deben ser abordadas como un dominio de resolución de problemas. Además, indica que hay cuatro habilidades o categorías de conocimiento, para que alguien sea bien sucedido en matemática, que son: i) Recursos, que son conocimientos de procedimientos y temas de matemática; ii) Heurística, que son estrategias y técnicas para resolver problemas, tales como trabajar con lo que le fue enseñado, así como montar esquemas y dibujar figuras; iii) Control, que es decisión sobre cuándo y cuáles recursos usar en determinada situación; iv) Convicciones, que es una visión matemática del mundo, la cual determina cómo alguien abordará un problema. 
Kantowski (1980), dividió la capacidad de resolución de problemas en cuatro estadios, pero Pereira (2002) los amplió a cinco, resultando en los siguientes niveles de capacitación del que resuelve problemas: a) Inerte, que es la persona que no tiene ningún o casi ningún entendimiento de lo que sea resolver un problema matemático; o sea, no sabe por dónde empezar. Lo máximo que consigue es reproducir procedimientos muy simples, que fueron exhaustivamente explicados y ejemplificados; b) Imitador, es el que con poca explicación y ejemplificación, se torna capaz de resolver ejercicios de matemática, pero todavía no consigue resolver verdaderos problemas. Participa productivamente en grupos que estén discutiendo la resolución de problemas nuevos, pero es incapaz de trabajar solo; c) Capaz, es el que atingió la capacidad de resolver problemas, pero ésos deben ser variantes relativamente simples de problemas que aprendió o que ya resolvió; d) Avanzado, es el que demuestra una capacidad superior de resolución de problemas, a través de la mayor velocidad de resolución en relación a otras personas, de la mayor complejidad de los problemas que es capaz de enfrentar y además puede concebir procesos de resolución diferentes de los que había aprendido; y e) Artista, es la persona que no sólo atingió un grado superior de inventar nuevos procesos de resolución de problemas, así como se preocupa en explorar caminos diferentes de los ya recurridos y en buscar resoluciones elegantes o poderosas.

El enfoque de este trabajo es plantear el enunciado de un problema a partir de su solución, pero se puede usar ideas puestas aquí para resolver problemas reales, donde no hay enunciado, pero sólo hay un problema, como el presentado a continuación. Por ejemplo, alguien entra a trabajar en una destilería, sospecha que la torre de destilación no está funcionando bien (detecta el problema), debe formular el problema (crear el enunciado) y para ello debe recolectar información. Ese proceso demanda tiempo y dinero (ensayos, instrumentos de medición, revisión de la literatura etc.), así que debe realizar un planteo eficiente del enunciado que no demande demasiada información, pero a la vez que no deje nada importante sin cubrir. Además, debe considerar que parte de la información que conseguirá será incompleta, sino errónea. Para construir un enunciado correcto, el profesional debe conocer las variables principales del sistema que está estudiando; para ello elaborará un modelo mental del proceso de destilación. Con el enunciado establecido, pasará a plantear el modelo matemático; en esta etapa podrá descubrir que aún le falta información, y retrocederá para completar el enunciado. Nuevamente, él descubrirá que debe modificar el enunciado. Al finalizar todo el proceso, ese ingeniero sabrá bien lo que hizo y por qué lo hizo; y estará en condiciones de mejorar el proceso. Cuando se reemplace a ese profesional por otro, éste se encontrará con lo ya hecho y podrá mejorarlo, por ejemplo, repitiendo todo el trabajo mental que llevó a cabo el ingeniero original.

\section{IDEAS PARA EMPLEAR EN EL ANÁLISIS}

Sea una situación de matemática elemental, en la cual el profesor presenta en el pizarrón varios cálculos de raíz cuadrada. En la primera situación escribe:

$\sqrt[2]{10.000}=\sqrt[2]{100 \times 100}=\sqrt[2]{100^{2}}=100$

Y pregunta ¿cuál es el significado de las operaciones matemáticas realizadas? Una respuesta sencilla es: la raíz cuadrada de 10.000 es igual a la raíz cuadrada de 100 veces 100 , que es igual a la raíz de 100 al cuadrado, que vale 100. Ya para el siguiente ejemplo la respuesta puede ser diferente. Sea:

$$
\sqrt[2]{(100 m)^{2}+(100 m)^{2}}=\sqrt[2]{20.000 m^{2}}=141,4 m
$$

Una posible respuesta es: la raíz cuadrada de 100 metros al cuadrado más 100 metros al cuadrado es $141,4 \mathrm{~m}$. Otro razonamiento es: la hipotenusa de un triángulo equilátero de lado 100 $\mathrm{m}$ es igual a $141,4 \mathrm{~m}$. O bien, la mínima distancia que una persona necesita caminar para atravesar una plaza, de $100 \mathrm{~m}$ de lado, es de 141,4 m. Por lo tanto, con análisis relativamente sencillos se puede obtener el planteo de muchos problemas, partiendo de su solución o de sus consecuencias. Lo principal que se discute aquí son ideas para ayudar en el análisis de una solución de un problema para encontrar su enunciado, más que una metodología general de análisis. Es decir, lo usual es que se enuncie y resuelva un problema, pero no es frecuente que se presente una solución de un problema y se pida hallar su enunciado. Aunque en la práctica se 
trabaje con situaciones combinadas de planteo y solución de problemas o viceversa, sin que la mayoría se dé cuenta de ello. Para obtener el enunciado de un problema de matemática o física, en el presente trabajo, se propone el conjunto de etapas presentadas a continuación, las cuales son detalladas en la Tabla 2, en forma de un diagrama a seguir para estructurar el pensamiento: i) Realizar un análisis de la solución de un problema, tratando de obtener su enunciado; ii) Elaborar un plan para obtener un enunciado del problema a partir de su solución; iii) Ejecutar el plan para obtener un enunciado del problema; y iv) Realizar un análisis retrospectivo del enunciado y verificar si con él se obtiene la solución inicialmente planteada.

Tabla 2: Cómo obtener el enunciado de un problema de matemática o física, partiendo de su solución.

\begin{tabular}{|c|c|}
\hline Etapa & Procedimiento \\
\hline $\begin{array}{l}\text { i) Realizar un análisis de } \\
\text { la solución de un } \\
\text { problema, tratando de } \\
\text { obtener su enunciado. }\end{array}$ & $\begin{array}{l}\text { - Analizar la solución propuesta para el problema. ¿Qué se propone } \\
\text { con ella? Antes que todo, la parte verbal de la solución de un } \\
\text { problema debe ser bien comprendida. } \\
\text { - ¿La secuencia propuesta de solución tiene lógica? ¿Es posible } \\
\text { elaborar un primer enunciado del problema, partiendo de ésta } \\
\text { solución? } \\
\text { - ¿Es posible confeccionar una figura, un diagrama o un esquema, } \\
\text { con el que uno pueda visualizar el enunciado del problema, o sea, } \\
\text { ver la solución al revés? } \\
\text { - ¿Es posible suponer un enunciado para el problema resuelto? }\end{array}$ \\
\hline $\begin{array}{l}\text { Elaborar un plan para } \\
\text { obtener un enunciado } \\
\text { del problema a partir } \\
\text { de su solución. }\end{array}$ & $\begin{array}{l}\text { - ¿Cuál es el plan para obtener el enunciado del problema? Lo } \\
\text { principal para obtener el enunciado de un problema es la } \\
\text { elaboración de un plan de acción. } \\
\text { - ¿Qué estrategias pueden ser desarrolladas para encontrar un } \\
\text { enunciado? } \\
\text { - ¿Hay algún enunciado semejante, que pueda ayudar a enunciar } \\
\text { éste? } \\
\text { - Acordarse que lo indispensable para obtener el enunciado de un } \\
\text { problema es el conocimiento adquirido, por ejemplo, a través de } \\
\text { enunciados anteriormente planteados. }\end{array}$ \\
\hline $\begin{array}{l}\text { iii) Ejecutar el plan para } \\
\text { obtener un enunciado } \\
\text { del problema. }\end{array}$ & $\begin{array}{l}\text { - Ejecutar el plan elaborado, verificándolo paso a paso. } \\
\text { - Rever la teoría correspondiente al problema resuelto, para auxiliar } \\
\text { en la obtención de un enunciado del problema. } \\
\text { - El plan es sólo un trayecto general para obtener el enunciado, pero } \\
\text { se necesita estar convicto de que todos los pasos son correctos. } \\
\text { Por eso hay que examinarlos, uno tras otro, pacientemente, hasta } \\
\text { que todo esté perfectamente claro y que no exista ningún punto } \\
\text { nebuloso en el cual pueda ocultarse algún error. } \\
\text { - Intentar ordenar los dados en tablas, diagramas o esquemas. } \\
\text { - Tratar de armar el enunciado del problema por partes. } \\
\text { - Efectuar los cálculos indicados en el plan, repitiendo cálculos ya } \\
\text { realizados en la solución del problema, para encontrar posibles } \\
\text { errores de cálculo o metodológicos. } \\
\text { - Realizar todas las estrategias pensadas en el plan, tratando de } \\
\text { obtener varios enunciados para el problema. }\end{array}$ \\
\hline $\begin{array}{l}\text { Realizar un análisis } \\
\text { retrospectivo del } \\
\text { enunciado y verificar si } \\
\text { con él se obtiene la } \\
\text { solución inicialmente } \\
\text { planteada. }\end{array}$ & $\begin{array}{l}\text { - ¿Es posible saber si el enunciado es correcto? } \\
\text { - Un análisis retrospectivo del enunciado, reexaminando el resultado } \\
\text { final y el camino que condujo a éste, puede ayudar a consolidar } \\
\text { conocimientos adquiridos y la habilidad para enunciar problemas. } \\
\text { - Verificar si el enunciado está correcto. Rever retrospectivamente la } \\
\text { solución del problema, contrastándola con el enunciado, buscando } \\
\text { posibles fallas. } \\
\text { - Conferir los cálculos realizados para llegar al enunciado, buscando } \\
\text { posibles errores en la metodología de cálculo o en la manera de } \\
\text { plantear el enunciado a partir de su solución. } \\
\text { - ¿Hay otra u otras maneras o métodos para encontrar el enunciado? } \\
\text { - ¿Es posible usar éste método para enunciar otros problemas o } \\
\text { semejantes, a partir de su solución? }\end{array}$ \\
\hline
\end{tabular}


Las ideas presentadas en las etapas (i) a (iv), de la Tabla 2, son sólo guías para obtener un enunciado de un problema, pero no garantizan que se pueda obtener un enunciado y tampoco que él sea único. Hay similitud entre las etapas propuestas en este trabajo y las presentadas por Polya (1995), pero existe una diferencia importante, es decir, Polya propone resolver problemas y en el presente artículo se trata de encontrar el enunciado de un problema resuelto. Lo que se propone aquí es que cuando uno tenga una solución de un problema, un accidente o un fenómeno inusitado, se transforme en habitual el recurrir al análisis, dando marcha atrás, hacia adelante o dando vueltas a la solución, para conocer y plantear el enunciado de un problema; una metodología que también puede ser válida para resolver un enigma. No hay reglas fijas, pero si es un problema de ingeniería, las bases son la física y matemática, y si es de otra área, deben usarse los conocimientos adecuados correspondientes. En último análisis, lo que se pretende es ofrecer nuevas perspectivas o estrategias que estimulen el análisis crítico y el razonamiento creativo, tratando de plantear un enunciado de un problema a partir de su solución, encontrar los motivos de fallas en equipos y procesos o dilucidar un enigma.

\section{RESULTADOS Y DISCUSIÓN}

A continuación se presenta la solución de un problema de intercambiadores de calor, que formó parte de un examen para estudiantes de ingeniería eléctrica, de materiales y computación. En el inicio se hacen algunas consideraciones, que fueron presentadas por escrito a los estudiantes; posteriormente se presenta una solución del problema; después se muestran los planteos del problema realizados por los estudiantes; y finalmente se discute cómo obtener algunas ecuaciones, para realizar simulaciones y calcular parámetros. El ejemplo resuelto está basado en un problema propuesto en el libro de Heldman y Singh (1981). Se puede hallar una discusión sobre las ecuaciones básicas de proyecto de intercambiadores de doble tubo en varios libros (Bird et al., 2002; Heldman y Singh, 1981). El objetivo del ejemplo presentado es mostrar una manera de analizar la solución de un problema sencillo, alentando a que otras personas desarrollen análisis más elaborados para obtener el planteo de un problema cualquiera.

\section{Enunciado del Examen}

Como se expuso en clase, se puede partir de una solución de un problema, para intentar encontrar su enunciado. En realidad, puede existir más de un planteo para un problema, así como algunos problemas tienen más de una solución. Partiendo de una solución de un problema de intercambiadores de calor, presentada a continuación, proponga un enunciado para el problema. Se pueden elaborar esquemas iniciales, pero enseguida debe obtenerse el enunciado completo del problema. Después de tener el planteo completo, es importante probar si, a partir de él, es posible obtener la solución propuesta aquí.

En una planta se tiene el intercambiador $\mathrm{A}$, con coeficiente global de intercambio de calor $\mathrm{Ua}=$ $500 \mathrm{Wm}-20 \mathrm{C}-\mathrm{l}$ y área de intercambio $\mathrm{Aa}=0,4 \mathrm{~m} 2$, y el intercambiador $\mathrm{B}$, con $\mathrm{Ub}=300 \mathrm{Wm}-2 \mathrm{oC}-1$ y $A b=1,0 \mathrm{~m} 2$. Las condiciones de proceso en un intercambiador genérico (i) para el alimento líquido, son velocidad másica de flujo $\mathrm{wf}=200 \mathrm{~kg} / \mathrm{h}$, calor específico Cpf $=3.600 \mathrm{Jkg}-1 \mathrm{oC}-1$, temperatura de entrada y salida Tf1 $=30{ }^{\circ} \mathrm{C}$ y Tf2 $=90{ }^{\circ} \mathrm{C}$, respectivamente. Para el aceite los parámetros son $w q=200 \mathrm{~kg} / \mathrm{h}, \mathrm{Cpq}=2.100 \mathrm{Jkg}-1 \mathrm{oC}-1$ y Tq2 $=160{ }^{\circ} \mathrm{C}$. A partir de ecuaciones básicas de un intercambiador de doble tubo, se obtiene para el fluido frío, que la potencia intercambiada $\mathrm{Qf}(\mathrm{J} / \mathrm{s})$ es:

$$
Q_{f}=w_{f} C p_{f}\left(T_{f 2}-T_{f 1}\right)
$$

Reemplazando los valores presentados en la Ec.(1), resulta en $\mathrm{Qf}=12.000 \mathrm{~W}$. Si no hay pérdidas de energía para el ambiente, la potencia cedida por el fluido caliente $\mathrm{Qq}(\mathrm{W})$ es igual a la recibida por el fluido frío $\mathrm{Qf}(\mathrm{W})$, es decir:

$$
Q_{q}=-Q_{f}=w_{q} C p_{q}\left(T_{q 1}-T_{q 2}\right)
$$


A partir de la Ec.(2) se obtiene que $\mathrm{Tq} 1=57,1{ }^{\circ} \mathrm{C}$. Para calcular el producto UA, puede ser empleada la ecuación presentada a continuación:

$$
\mathrm{Q}_{\mathrm{f}}=\frac{\operatorname{UA}\left\{\left(\mathrm{T}_{\mathrm{q} 2}-\mathrm{T}_{\mathrm{f} 2}\right)-\left(\mathrm{T}_{\mathrm{q} 1}-\mathrm{T}_{\mathrm{f} 1}\right)\right\}}{\ln \left\{\left(\mathrm{T}_{\mathrm{q} 2}-\mathrm{T}_{\mathrm{f} 2}\right) /\left(\mathrm{T}_{\mathrm{q} 1}-\mathrm{T}_{\mathrm{f} 1}\right)\right\}}
$$

De la Ec.(3) se calcula UiAi $=295,6 \mathrm{~W} / \mathrm{oC}$, para el intercambiador genérico (i), con Qf $=12.000 \mathrm{~W}$ y las temperaturas mencionadas. Comparando el intercambiador (i) $\mathrm{UiAi}=295,6 \mathrm{~W} / \mathrm{oC}$, con $\mathrm{A}$ y $\mathrm{B}$, respectivamente, UaAa $=500 \mathrm{Wm}-20 \mathrm{C}-1(0,4 \mathrm{~m} 2)=200 \mathrm{~W} / \mathrm{oC}$ y UbAb $=300 \mathrm{Wm}-2 \mathrm{oC}-1(1 \mathrm{~m} 2)=$ $300 \mathrm{~W} / \mathrm{oC}$, se selecciona uno, ninguno o ambos intercambiadores. Es decir, el intercambiador escogido debe aportar por lo menos $Q f=12.000 \mathrm{~W}$. Usando UaAa $=200 \mathrm{~W} / \mathrm{oC}$ y las temperaturas de entrada y salida para el intercambiador $A$, resulta en $\mathrm{Qa}=9.047,5 \mathrm{~W}$. Con UbAb $=200 \mathrm{~W} / \mathrm{oC}$, para el intercambiador $\mathrm{B}$, resulta en $\mathrm{Qb}=13.571,2 \mathrm{~W}$. Por lo tanto, el intercambiador seleccionado es $\mathrm{B}$, porque la potencia $\mathrm{Qb}=13.571,2 \mathrm{~W}>12.000 \mathrm{~W}$; pero el intercambiador $\mathrm{A}$, con $\mathrm{Qa}=9.047,5$ W, no aporta potencia suficiente para el proceso, aunque dispusiera de iguales temperaturas $\mathrm{Tq}$ y Tf, tanto de entrada como de salida que el intercambiador B. A continuación se presentan propuestas de planteo del problema de intercambiadores de doble tubo, realizadas por tres grupos de estudiantes y el enunciado del libro de Heldman y Singh (1981).

\section{Planteo del Grupo 1}

Analizando un intercambiador de calor de doble tubo, el calor se transmite a través de la pared del tubo interno; las corrientes de fluido circulan en contracorriente. Suponiendo que el flujo de calor es en estado estacionario y que la pérdida de calor hacia el entorno es despreciable, están disponibles el intercambiador $\mathrm{A}$ : $\mathrm{Ua}=500 \mathrm{Wm}-2 \mathrm{oC}-1$ y $\mathrm{Aa}=0,4 \mathrm{~m} 2$, y el intercambiador $\mathrm{B}: \mathrm{Ub}=$ $300 \mathrm{Wm}-20 \mathrm{C}-1$ y $\mathrm{Ab}=1,0 \mathrm{~m} 2$. ¿Cuál sería el intercambiador ideal para calentar un alimento líquido, usando aceite como fluido caliente, en contracorriente? (Además, el grupo presentó un esquema de un intercambiador en contracorriente, incluyendo las temperaturas de entrada, salida, datos del alimento $w f=200 \mathrm{~kg} / \mathrm{h}$ y Cpf $=3.600 \mathrm{Jkg}-10 \mathrm{C}-1$, y del aceite $w q=200 \mathrm{~kg} / \mathrm{h}$ y Cpq $=$ $2.100 \mathrm{Jkg}-10 \mathrm{C}-1$.)

\section{Planteo del Grupo 2}

Queremos comprar un intercambiador de calor de doble tubo, para ser usado en contracorriente, en el problema enunciado a continuación: enfriar un alimento líquido, que pasa por el tubo interno, con aceite, que pasa por el tubo externo. Las especificaciones del problema son presentadas en la siguiente tabla.

\begin{tabular}{|l|l|l|l|l|}
\hline Material & $\begin{array}{l}\text { Velocidad másica } \\
(\mathrm{kg} / \mathrm{h})\end{array}$ & $\begin{array}{l}\text { Calor específico } \\
(\mathrm{Jkg}-10 \mathrm{O}-1)\end{array}$ & $\begin{array}{l}\mathrm{T}_{\text {entrada }} \\
\left({ }^{\circ} \mathrm{C}\right)\end{array}$ & $\begin{array}{l}\mathrm{T}_{\text {salida }} \\
\left({ }^{\circ} \mathrm{C}\right)\end{array}$ \\
\hline Alimento líquido & 200 & 3.600 & 90 & 30 \\
\hline Aceite & 200 & 2.100 & ---- & 160 \\
\hline
\end{tabular}

Una empresa tiene disponibles dos intercambiadores. El intercambiador A especificado con coeficiente global $\mathrm{Ua}=500 \mathrm{Wm}-2 \mathrm{oC}-1$ y área para intercambio de calor $\mathrm{Aa}=0,4 \mathrm{~m} 2$. El intercambiador $\mathrm{B}$ con $\mathrm{Ub}=300 \mathrm{Wm}-20 \mathrm{C}-1$ y $\mathrm{Ab}=1,0 \mathrm{~m} 2$. ¿Cuál intercambiador sería el adecuado para resolver nuestro problema (enfriar el alimento líquido hasta $30^{\circ} \mathrm{C}$ ), el $\mathrm{A}$, el $\mathrm{B}$ o ninguno de ellos?

\section{Planteo del Grupo 3}

En determinada industria se dispone de dos intercambiadores de calor de tubos concéntricos, con configuración en contracorriente; teniendo el intercambiador $\mathrm{A}$ coeficiente global de transferencia de calor $\mathrm{Ua}=500 \mathrm{Wm}-2 \mathrm{oC}-1$ y área $\mathrm{Aa}=0,4 \mathrm{~m} 2$, y el intercambiador $\mathrm{B}, \mathrm{Ub}=300 \mathrm{Wm}-2 \mathrm{oC}-1$ y $\mathrm{Ab}$ $=1,0 \mathrm{~m} 2$. Se necesita seleccionar uno de los dos intercambiadores, de modo que aporte potencia suficiente para enfriar aceite lubricante de un motor, con temperatura $\mathrm{Tq} 2=160{ }^{\circ} \mathrm{C}$ y calor específico $\mathrm{Cpq}=2.100 \mathrm{Jkg}-1 \mathrm{oC}-1$. Se utilizará un alimento líquido en las siguientes condiciones: 
$\mathrm{Tf} 2=90{ }^{\circ} \mathrm{C}, \mathrm{Tf} 1=30{ }^{\circ} \mathrm{C}$ y $\mathrm{Cpf}=3.600 \mathrm{Jkg}-1 \mathrm{oC}-1$. Si la velocidad másica de ambos fluidos es wf $=$ $200 \mathrm{~kg} / \mathrm{h}$, determine la potencia mínima de funcionamiento y con base en el resultado, ¿cuál intercambiador será utilizado?

\section{Planteo del Problema en el Libro de Heldman y Singh (1981)}

Un alimento líquido, con calor específico $\mathrm{Cp}=3.600 \mathrm{Jkg}-1 \mathrm{oC}-1$ va a ser calentado de 30 a $90{ }^{\circ} \mathrm{C}$. El medio de calentamiento es aceite, con $\mathrm{Cp}=2.100 \mathrm{Jkg}-1 \mathrm{oC}-1$, entrando a la temperatura de 160 ${ }^{\circ} \mathrm{C}$. La velocidad másica del aceite y del alimento líquido es $w=200 \mathrm{~kg} / \mathrm{h}$. Dos intercambiadores de calor de doble tubo están disponibles. El intercambiador A tiene un coeficiente global de transferencia de calor $U=500 \mathrm{Wm}-2 \mathrm{oC}-1$ con área total de $0,4 \mathrm{~m} 2$ y el intercambiador $\mathrm{B}$ tiene $\mathrm{U}=$ $300 \mathrm{Wm}-20 \mathrm{C}-1$ y área total de $1 \mathrm{~m} 2$. ¿Cuál de los intercambiadores debe seleccionarse para esta aplicación?

Nótese que los enunciados presentados por los estudiantes son similares, pero difieren principalmente en la manera como cada grupo enfrenta la solución del problema, para proponer su planteo. Por ejemplo, en el primer planteo hay preocupaciones y descripciones teóricas, pero en el segundo y tercero, se destacan consideraciones prácticas. El segundo grupo considera que el alimento líquido es enfriado, al contrario de lo enunciado en el libro de Heldman y Singh (1981), es decir, el alimento es calentado. Esos distintos análisis tal vez tengan que ver con las diferentes posibilidades con que se puede visualizar un problema, con el tipo de formación promedio de cada grupo, con sus preocupaciones y quizás con la personalidad promedio grupal o con la dominante. Se concluye que por lo general los estudiantes sortearon bien este examen y que propuestas como la presentada ayudan a retroalimentar conocimientos, que es una manera complementaria de visualizar y bucear en soluciones y planteos de problemas. Ésas metodologías estimulan el razonamiento, amplían el conocimiento y la habilidad para analizar problemas, dando marcha atrás o hacia delante. Pero los estudiantes no pusieron atención en rehacer los cálculos presentados con anterioridad, para buscar posibles errores en los cálculos desarrollados por el docente. A continuación son realizados nuevamente algunos cálculos y discutidas otras posibilidades de selección de los intercambiadores. Hay error de cálculo en el parámetro AiUi presentado por el docente, lo que produjo errores en cálculos posteriores; por eso AiUi es recalculado, ampliando el análisis, sin necesidad de incluir errata en el texto. De la Ec.(3) se calcula UiAi $=265,3 \mathrm{~W} / \mathrm{oC}$, con Tq1 $=57,1^{\circ} \mathrm{C}, \mathrm{Tf} 1=30{ }^{\circ} \mathrm{C}, \mathrm{Tf} 2=90{ }^{\circ} \mathrm{C}, \mathrm{Tq} 2=160{ }^{\circ} \mathrm{C}$ y Qf $=$ $12.000 \mathrm{~W}$. Comparando el intercambiador (i) UiAi $=265,3 \mathrm{~W} / \mathrm{oC}$, con $\mathrm{A}$ y $\mathrm{B}, \mathrm{UaAa}=200 \mathrm{~W} / \mathrm{oC}$ y $\mathrm{UbAb}=300 \mathrm{~W} / \mathrm{oC}$, se selecciona el intercambiador $\mathrm{B}$, pues $\mathrm{Qb}=13.571,2 \mathrm{~W}>12.000 \mathrm{~W}$, que coincide con el seleccionado con anterioridad; pero el intercambiador $\mathrm{A}$, con $\mathrm{Qa}=9.047,5 \mathrm{~W}$, no aporta potencia suficiente para el proceso.

Aun siendo adecuada la selección del intercambiador $B$, hay que imponer otros criterios en tal selección. Es decir, partiendo de $\mathrm{Qb}=13.571,2 \mathrm{~W}$, si fueren mantenidas ambas velocidades másicas en $\mathrm{w}=200 \mathrm{~kg} / \mathrm{h}$, las temperaturas de salida de las dos corrientes serán diferentes de las calculadas con anterioridad. Como no fueron presentadas informaciones sobre la resistencia del alimento líquido a $\mathrm{T}>90^{\circ} \mathrm{C}$, quizás sea adecuado seleccionar otra wf para el alimento, para que él continúe entrando a $90^{\circ} \mathrm{C}$ y saliendo a $30^{\circ} \mathrm{C}$. En estas condiciones, con $\mathrm{Qf}=13.571,2 \mathrm{~W}, \mathrm{Cpf}=$ $3.600 \mathrm{Jkg}-10 \mathrm{C}-1, \mathrm{Tf} 1=30^{\circ} \mathrm{C}$ y Tf2 $=90^{\circ} \mathrm{C}$, de la Ec.(1) se obtiene la nueva velocidad másica para el alimento wf2 $=226,2 \mathrm{~kg} / \mathrm{h}$. Como wf aumentó de wf1 $=200 \mathrm{~kg} / \mathrm{h}$ para wf2 $=226,2 \mathrm{~kg} / \mathrm{h}$, bajo las mismas condiciones de operación de la corriente fría, entonces el coeficiente global $U$ debe cambiar. Por lo tanto, la discusión presentada a continuación es realizada para poder calcular los nuevos valores de Tq1, U2 y wq2.

Se evalúa el coeficiente $U$, de la Ec.(3), despreciando las resistencias de incrustación, la resistencia térmica de la pared, admitiendo que la pared del tubo interno tiene áreas de intercambio de calor interna y externa casi iguales, y conociéndose el valor del coeficiente convectivo interno al tubo interno hint(Wm-2oC-1) y externo al tubo interno hext(Wm-2oC-1), empleando la ecuación presentada a continuación (Bird et al., 2002; Heldman y Singh, 1981; Incropera y De Witt, 1998):

$$
U \cong \frac{1}{1 / h_{\text {int }}+1 / h_{\text {ext }}}
$$


Para flujo laminar de fluidos en tubos de diámetro $D(m)$, es decir, para número de Reynolds $\operatorname{Re}<$ 2.100, puede ser empleada la ecuación presentada a continuación (Heldman y Singh, 1981; Incropera y De Witt, 1998), para evaluar el coeficiente convectivo de transferencia de calor h(Wm2oC-1):

$$
\frac{\mathrm{hD}}{\mathrm{k}}=1,86\left(\frac{\mathrm{wCp}}{\mathrm{kL}}\right)^{1 / 3}\left(\frac{\mu}{\mu_{\mathrm{p}}}\right)^{0,14}
$$

El primer miembro de la Ec.(5) es el número de Nusselt Nu, el primer término entre paréntesis del segundo miembro es el número de Graetz Gz y $\mu \mathrm{p}(\mathrm{kgm}-1 \mathrm{~s}-1)$ es la viscosidad del fluido, evaluada a la temperatura de la pared del tubo. Para flujo turbulento en tubos, puede ser empleada la ecuación propuesta por Dittus y Boelter (1930), citada en varios libros (Heldman y Singh, 1981; Incropera y De Witt, 1998), para calcular el coeficiente convectivo de transferencia de calor h(Wm2oC-1):

$$
\frac{\mathrm{hD}}{\mathrm{k}}=0,023\left(\frac{\rho \mathrm{vD}}{\mu}\right)^{0,8}\left(\frac{\mu \mathrm{Cp}}{\mathrm{k}}\right)^{\mathrm{n}}
$$

El primero y segundo términos entre paréntesis del segundo miembro de la Ec.(6) son, respectivamente, el número de Reynolds Re y Prandtl $\mathrm{Pr}$. Si el exponente del número de Prandtl es $n=0,4$, significa que el fluido está siendo calentado y si es $n=0,3$, él está siendo enfriado. Las propiedades del fluido, evaluadas a la temperatura promedio del fluido, son calor específico $\mathrm{Cp}(\mathrm{Jkg}-10 \mathrm{C}-1)$, conductividad térmica $\mathrm{k}(\mathrm{Wm}-10 \mathrm{C}-1)$, densidad $\rho(\mathrm{kgm}-3)$ y viscosidad $\mu(\mathrm{kgm}-1 \mathrm{~s}-1)$. La Ec.(6) es válida para Reynolds $\operatorname{Re}>10.000$, el cual es función del área de la sección transversal del tubo Atra $(\mathrm{m} 2)$, del diámetro interno $\operatorname{Dint}(\mathrm{m})$, de la velocidad másica $\mathrm{w}(\mathrm{kg} / \mathrm{s})$ y densidad $\rho(\mathrm{kgm}-3)$. Para el flujo en el interior del tubo interno de un intercambiador de doble tubo, las ecuaciones de Re y w en el área interna, para emplear con la Ec.(5) o (6), son:

$$
\begin{aligned}
& \operatorname{Re}_{\text {int }}=\frac{\rho v D_{\text {int }}}{\mu}=\frac{4 w_{\text {int }}}{\pi \mu D_{\text {int }}} \\
& w_{\text {int }}=\rho v A_{\text {tra }}=\rho v \pi \frac{D_{\text {int }}{ }^{2}}{4}
\end{aligned}
$$

Las ecuaciones de Re y w en el área anular, para cálculos con la Ec.(5) o (6), para flujo en el área entre el tubo externo y el interno, es decir, en la región anular del intercambiador, despreciando el espesor de la pared del tubo interno, son:

$$
\begin{aligned}
& R e_{\text {anu }}=\frac{\rho v\left(D_{\text {ext }}-D_{\text {int }}\right)}{\mu}=\frac{4 w_{\text {anu }}}{\pi \mu\left(D_{\text {ext }}+D_{\text {int }}\right)} \\
& w_{\text {anu }}=\rho v A_{\text {anu }}=\rho v \frac{\pi}{4}\left(D_{\text {ext }}^{2}-D_{\text {int }}^{2}\right)
\end{aligned}
$$

En las Ec.(9) y (10) el término $D=$ (Dext - Dint) es el diámetro hidráulico del espacio anular y Dext es el diámetro interno del tubo externo. El diámetro hidráulico puede ser empleado tanto en la Ec.(5) como en la Ec.(6). Si hay un cambio de wq1 para wq2, para el fluido caliente y de wf1 para wf2, para el frío, se evalúa el nuevo h2, a partir del coeficiente inicial h1, admitiendo que las propiedades de los fluidos no se modifican en el rango de temperatura considerado. Si la Ec.(5) es válida para el fluido caliente y la Ec.(6) para el frío, empleando dos veces cada una de ellas, resulta en:

$$
\begin{aligned}
& \mathrm{h}_{\mathrm{q} 2}=\mathrm{h}_{\mathrm{q} 1}\left(\mathrm{w}_{\mathrm{q} 2} / \mathrm{w}_{\mathrm{q} 1}\right)^{1 / 3} \\
& \mathrm{~h}_{\mathrm{f} 2}=\mathrm{h}_{\mathrm{f} 1}\left(\mathrm{w}_{\mathrm{f} 2} / \mathrm{w}_{\mathrm{f} 1}\right)^{0,8}
\end{aligned}
$$


Reemplazando en la Ec.(4) los nuevos valores de h2, para el aceite h2q y alimento h2f, y la nueva wf2 $=226,2 \mathrm{~kg} / \mathrm{h}$, se obtiene la ecuación de cálculo del nuevo U2, en la cual hq1 y hf1 son evaluados por la Ec.(5) y (6), respectivamente, con velocidades másicas iniciales wq1 = wf1 $=200$ $\mathrm{kg} / \mathrm{h}$ :

$$
\mathrm{U}_{2} \cong \frac{1}{\frac{1}{\mathrm{~h}_{\mathrm{q} 1}\left(\mathrm{w}_{\mathrm{q} 2} / \mathrm{w}_{\mathrm{q} 1}\right)^{1 / 3}}+\frac{1}{\mathrm{~h}_{\mathrm{f} 1}\left(\mathrm{w}_{\mathrm{f} 2} / \mathrm{w}_{\mathrm{f} 1}\right)^{0,8}}}
$$

Realizando simulaciones con las Ecs.(13), (1), (2) y (3), se obtienen los nuevos valores de Tq1, $\mathrm{U} 2$ y wq2, partiendo del dato de proyecto $\mathrm{Qf}=13.571,24 \mathrm{~W}$; pues ya fue seleccionada wf2 $=226,2$ $\mathrm{kg} / \mathrm{h}$ etc.

\section{CONCLUSIONES}

Se concluye que el diagrama propuesto para obtener el enunciado de un problema basándose en su solución, puede ser empleado, por ejemplo, para enunciar problemas de matemática, física, ingeniería y áreas relacionadas; pudiendo ser adaptado para su empleo en otras áreas.

Las ideas de análisis propuestas en este artículo ayudan a retroalimentar conocimientos y representan maneras complementarias a otras bien conocidas, para visualizar, ojear y bucear en soluciones y enunciados de problemas. Ésas metodologías estimulan el razonamiento, amplían el conocimiento y la habilidad para analizar soluciones o resolver problemas, dando marcha atrás o hacia delante.

Esquemas como el presentado ayudan a organizar ideas y estructurar el pensamiento, para enunciar problemas partiendo de su solución; tal vez mejorando la creatividad, la enseñanza y el aprendizaje, y quizás posibiliten la descubierta de nuevos caminos en investigación y actividades cotidianas.

\section{REFERENCIAS}

Bird, R.B., W.E. Stewart y E.N. Lightfoot; Transport Phenomena, p.307, 425 y 462-463, John Wiley, New York, USA (2002).

Dante, L.R.; Didáctica de Resolución de Problemas de Matemática, p.29, Ática, São Paulo (2002). (En Portugués.)

Heldman, D.R. y R.P. Singh; Food Process Engineering, p.94-96, 100, 117 y 147, AVI Publishing Company, Westport, Connecticut (1981).

Incropera, F.K., D.P. De Witt; Fundamentos de Transferencia de Calor y Materia, p.50, 240, 249, 320 y 463, Livros Técnicos e Científicos, Rio de Janeiro (1998). (En Portugués.)

Kantowski, M.G.; Some Thoughts on Teaching for Problem Solving, In Problem Solving in School Mathematics, Robert E. Reys (Ed.), Open University, London (1980).

Lima, J.L. y S.H. Massino; Manual de Detección de Fallas de una Planta Piloto de Producción de Quesos Basado en Conocimiento Experto, Información Tecnológica: 19(3), 65-74 (2008).

Nurminen, J., O. Karonen y K. Hätönen; What Makes Expert Systems Survive Over 10 Years, Expert Systems with Applications: 24 (2), 199-211 (2003).

Pereira, A.L.; Problemas Matemáticos: Caracterización, Importancia y Estrategias de Resolución, p.10 y 11-14, MAT450 - Seminarios de Resolución de Problemas, IME - USP - Instituto de Matemática y Estadística de la Universidad de São Paulo (2002). (En Portugués.)

Polya, G.; El Arte de Resolver Problemas: un Nuevo Aspecto del Método Matemático, p.XII-XIII, 16 y 24-27, Interciências, Rio de Janeiro (1995). (En Portugués.)

Schoenfeld, A.; Mathematical Problem Solving, Academic Press (1985).

Tarifa, E.E. y S.L. Martínez; Diagnóstico de Fallas con Redes Neuronales. Parte 1: Reconocimiento de Trayectorias, Revista Ingeniería e Investigación: 27(1), 68-76 (2007). 